\title{
Schule: global - postkolonial. Annäherungen an einen Zusammenhang
}

„Globales lehren, Postkoloniales lehren. Perspektiven für Schule im Horizont der Gegenwart" war der Titel einer internationalen Konferenz, die im Juli 2019 an der Friedrich-Schiller-Universität stattgefunden hat. Die Konferenz wurde in Kooperation zweier Graduiertenschulen der Universität Jena organisiert. Eingeladen hatten das am Institut für Bildung und Kultur angesiedelte „Kolleg Globale Bildung“ sowie das Forschungs- und Doktorandenkolleg „Bildung. Forschung. Dialog.“, welches im Rahmen der Qualitätsoffensive Lehrerbildung etabliert wurde.

Zu den Eigenheiten der Lehramtsausbildung gehört, dass angehende Lehrkräfte in der Regel für einen konkreten nationalen bzw. regionalen Kontext ausgebildet werden. Gleichzeitig sind das Lehren und Lernen in der Schule und im Alltag heute unausweichlich von einem Horizont bestimmt, der als global gekennzeichnet werden muss. Für Lehrkräfte wie für Schüler/-innen stellt sich gleichermaßen die Herausforderung, diese beiden Bezugsgrößen im eigenen Denken und Handeln zusammenzubringen. Die Spannung zwischen Regionalem und Globalem wird dadurch noch brisanter, dass postkoloniale Interventionen die Frage aufwerfen, auf welche Art und Weise unsere, über Lernen aufgebauten Vorstellungen von Normalität durch machtdurchsetzte Annahmen und Strukturen geprägt wird. Die (postkoloniale) Auseinandersetzung mit Marginalisierung, Stigmatisierung, Ausgrenzung oder Ausbeutung partikularer Gruppen und die Frage, was die damit verbundenen Perspektiven mit uns zu tun haben, stehen dabei auf der einen Seite. Diese Wahrnehmung von Differenz trifft auf der anderen Seite auf einen systematischen Zusammenhang globaler Herausforderungen, in dem nach einer gemeinsamen, „inklusiven“ Zukunft für Alle (angefangen im Programm „Education for all“ bis hin zu Praktiken nachhaltigen Handelns von Konsumsteuerung bis Klimawandel) gefragt wird. Globales und Postkoloniales erscheinen gerade vor dem Hintergrund, Kindern und Jugendlichen eine reflektiert-realistische Vorstellung von "Welt“ zu vermitteln, als feindlich-freundliche Geschwister, die zumindest auf den ersten Blick nur teilweise ähnliche oder gleiche Ziele verfolgen. Vor dem Hintergrund dieser Gemengelage hat die internationale Konferenz in aller Vorläufigkeit nach Impulsen dafür gesucht, was es bedeuten kann, (schulisches) Lehren und Lernen gleichzeitig vor dem Hintergrund globaler und postkolonialer Motive zu verstehen.

Gerahmt wurde die zweitägige Veranstaltung durch zwei Keynote-Vorträge, in denen das Verhältnis „Global - postkolonial. Über die Schulung paradoxer Weltwahrnehmung“ (Ralf Koerrenz, Jena) und eine „Bildung zur Nachhaltigkeit“ (Alex Lautensach, British Columbia/Kanada) skizziert wurden. Es folgten Vorträge zu Traditionslinien von Bildung, die mit dem Stichwort „global“ gekennzeichnet werden können (Lena Köhler, Jena), und zu der Problematik, dass von „Traditionen postkolonialer Bildung“ (Sebastian Engelmann, Tübingen) wenn überhaupt - nur im Modus einer kritischen Distanzierung vom Thema selbst gesprochen werden kann. Ein Versuch, sich dem aufscheinenden Spannungsverhältnis unterschiedlicher Rahmungen für das Verständnis von Lehren und Lernen zu nähern, bestand anschließend darin, die „Logik der Heterogenität" (Karsten Kenklies, Glasgow/Großbritannien) von der „Logik der Inklusion“ (Alexandra Schotte, Augsburg) zu unterscheiden. Eine besondere Bedeutung hat die Auseinandersetzung mit globalen und postkolonialen Horizonten für einen zeitgemäßen Geografieunterricht. Entsprechende Perspektiven wurden in Überlegungen zur „Welt im Werden. Zur Krise der Repräsentation“ (Mirka Dickel, Jena) und „Über Beispiele. Das Exemplarische und das Allgemeine im Globalen Lernen“" (Fabian Pettig, Jena) entwickelt.

Mit den USA und Kanada richtete die Konferenz die Aufmerksamkeit auf besonders konfliktreiche Sozialstrukturen. „Cultural Pluralism and Equity in US-American Education“ (Elizabeth Watts, Weimar/Jena) und „Cultural Pluralism and Equity in Canadian Education" (Sabina Lautensach, British Columbia/Kanada) warfen Schlaglichter auf Schule im Kontext von spezifischen gesellschaftlichen Zusammenhängen. Exemplarisch ergänzt wurden diese Beiträge durch eine Skizze „Pädagogische(r) Perspektiven im Anschluss an bell hooks“ (Milena Morosoli, Jena) und durch einen kritischen Blick auf das „Canadian Curriculum between Manifest Manners and Decolonization" (Kathryn Arsenault, Weimar).

Wenn wir Schule unter den Vorzeichen "global“ und „postkolonial“ betrachten wollen, so treffen wir mit Blick auf schulische Praxis auf eine dynamisch-offene Situation. Auch wenn beispielsweise Themen wie Rassismus und die systematisch-logischen Fallstricke von Kollektivzuschreibungen als Beispiele für postkoloniale Anliegen im schulischen Lehrplan Erwähnung finden, scheinen die Transformationen von Themen globalen Lernens in den schulischen Alltag nicht zuletzt über den „Orientierungsrahmen“ deutlich ausgereifter und verbreiteter. Neben diese Beobachtung gesellt sich die Frage nach den normativen Logiken der Didaktik zwischen Dekonstruktion von Macht- und Ausgrenzungsverhältnissen und konstruktiven Zukunftsgestaltungen. Allein in dem Titel eines Programms wie „Bildung trifft Entwicklung“ tut sich je nach globaler oder postkolonialer Optik ein unterschiedlicher normativer Bewertungszusammenhang auf, der das Nachdenken über das Verhältnis von "global - postkolonial“ mit Blick auf Schule nur in einer spannungsreichen Geschwisterlichkeit denkbar werden lässt. Die Jenaer Konferenz kann als ein Set fragmentarischer Denkanstöße zur Diskussion dieses Verhältnisses verstanden werden. Die Studien im Anschluss an die Konferenz sind unter dem Titel „Globales lehren - Postkoloniales lehren. Perspektiven für Schule im Horizont der Gegenwart“ im Beltz-Verlag (Weinheim, 2020) in der Reihe „Bildung: Demokratie“ erschienen.

Ralf Koerrenz

Friedrich-Schiller-Universität Jena doi.org/10.31244/zep.2020.03.10 